\title{
Correlation Between Calcification Characteristics of Carotid Atherosclerotic Plaque and Plaque Vulnerability
}

\author{
Xiangli $\mathrm{Xu}^{\mathrm{I}}$ \\ Yang Hua ${ }^{2}$ \\ Beibei Liu ${ }^{2}$ \\ Fubo Zhou ${ }^{2}$ \\ Lili Wang ${ }^{2}$ \\ Weihong $\mathrm{Hou}^{2}$
}

'Department of Ultrasound, the Second Hospital of Harbin, Harbin, People's Republic of China; ${ }^{2}$ Department of Vascular Ultrasonography, Xuanwu Hospital, Capital Medical University, Beijing, People's Republic of China
Correspondence: Yang Hua Department of Vascular Ultrasonography, Xuanwu Hospital, Capital Medical University, No. 45 Changchun Road, Beijing, 100053, People's Republic of China

Tel +86-10-83198923

$\mathrm{Fax}+86-10-83198967$

Email dryanghua99@163.com
Purpose: To investigate the relationship between calcification characteristics of carotid atherosclerotic plaque and lipid rich necrotic core (LRNC) and intraplaque hemorrhage (IPH).

Methods: Patients with severe carotid stenosis undergoing carotid endarterectomy (CEA) were selected. Ultrasound and CT angiography (CTA) were performed to evaluate the calcification characteristics of the plaque before the surgery.

Results: A total of 142 patients were included and 142 pathological specimens of postoperative plaque were obtained accordingly. There were 78 plaques $(54.9 \%)$ with LRNC and $41(28.9 \%)$ with IPH. The plaque with LRNC had higher calcification rate $(93.6 \%)$ compared with the plaque with IPH $(87.8 \%)$. LRNC was often found in multiple calcification $(\mathrm{P}=$ $0.003)$ and mixed type calcification $(\mathrm{P}=0.001)$. Multiple calcification was more likely to combine with IPH $(\mathrm{P}=0.008)$, while simple basal calcification was not likely to combine IPH $(\mathrm{P}=0.002)$. Smaller granular calcification was more likely to be associated with IPH (P $<0.05)$. In multivariate regression analysis of IPH and calcification characteristics, simple basal calcification was still a protective factor for IPH (OR, 0.25; 95\% CI, 0.09-0.66; P = $0.005)$, while multiple calcification was closely related to the occurrence of IPH (OR, 3.58; 95\% CI, 1.49-8.61; P = 0.004).

Conclusion: Calcification characteristics of carotid atherosclerotic plaques are closely related to the vulnerability of plaques, especially multiple calcification and mixed type calcification.

Keywords: carotid artery, atherosclerotic plaque, intraplaque calcification, vulnerable plaque

\section{Introduction}

Carotid atherosclerotic plaque is the main risk factor of ischemic cerebrovascular disease. ${ }^{1}$ The mechanisms of ischemic stroke induced by carotid atherosclerotic plaque are as follows: 1) severe carotid stenosis leads to distal hypoperfusion; 2) the thrombus shedding caused by plaque rupture causes artery blockage, which leads to the decrease of blood perfusion in the distal artery; 3) thrombosis secondary to plaque ulceration; 4) the volume of the plaque increases and the vascular lumen is occluded suddenly. ${ }^{2}$ Most previous studies have focused on the relationship between vascular stenosis and ischemic stroke. With the deepening of the study, it is found that cerebral infarction caused by carotid artery stenosis due to ischemia or hypoperfusion only accounts for a small part of clinical events, and most of them are vulnerable plaques (VP) rupture, secondary thrombosis and embolism, which lead to the occurrence of 
stroke. $^{3,4}$ Therefore, early identification of VP is very important to prevent ischemic cerebrovascular disease.

Plaque morphology components indicating plaque vulnerability are a thin or disrupted fibrous cap, a large lipid-rich necrotic core (LRNC), neovascular growth and intraplaque haemorrhage (IPH) ${ }^{5,6}$ whereas calcification is considered as a component of plaque stability. ${ }^{7}$ Many studies have also shown that the intraplaque IPH and LRNC are important predictors of plaque vulnerability, which will greatly increase the risk of stroke. ${ }^{8-10}$ However, the effect of other plaque components, especially calcification, on plaque vulnerability is still controversial. It has been reported that calcification is a "protective" factor of VP, which can stabilize plaque and greatly reduce the risk of stroke. ${ }^{11,12}$ In recent years, with the deepening of research and the progress of detection methods of calcified plaque, the results show that with the increase of the amount of calcification in the plaque, the probability of intra plaque hemorrhage may also increase, and some calcification or large volume calcification are more likely to be complicated with intra plaque hemorrhage, which is easy to cause plaque rupture. ${ }^{13-15}$ It has been shown that the stability of plaque cannot be evaluated simply based on the presence or absence of calcification, but more detailed observation of calcification features, such as calcification location, calcification type or calcification volume, etc., which will play a more important role in promoting the assessment of plaque vulnerability. ${ }^{9,16}$ Therefore, we should pay more attention to the composition of plaque, which may provide necessary information for the prevention and management of cerebrovascular diseases.

Computed tomographic angiography (CTA) provides valuable information not only about luminal stenosis but also about plaque characteristics. ${ }^{17}$ Therefore, in current study, LRNC and IPH of the carotid atherosclerotic plaque evaluated by histopathological examination and calcification characteristics using CTA and ultrasound were compared and influence of calcification characteristics on plaque vulnerability was investigated.

\section{Materials and Methods}

\section{Patients}

This is a retrospective study. From February 2016 to December 2016, the patients diagnosed with severe carotid stenosis, hospitalized in the Department of Vascular surgery or Neurosurgery of our hospital were continuously included. Inclusion criteria: All patients were diagnosed with severe carotid stenosis by color Doppler ultrasound (CDU). Computed tomography angiography (CTA) or digital subtraction angiography (DSA) was used to confirm the diagnosis of severe carotid stenosis. Carotid endarterectomy (CEA) was further performed in these patients. Culprit plaque of all patients was evaluated by CTA and ultrasonography within 48 hours before surgery. The exclusion criteria were: Patients have received carotid artery surgery or other special treatment, such as carotid artery stent implantation, carotid endarterectomy, long-term neck radiotherapy, etc. Patients with severe calcification or calcification accompanied by wide sound shadow cannot be evaluated. This study was approved by the Ethics Committee of the Xuanwu Hospital, Capital Medical University, Beijing, China. All patients gave informed written consent to participate in the study.

\section{Observation Index}

The basic clinical data include gender, age, body mass index (BMI), smoking status, hypertension, diabetes, hyperlipidemia and related laboratory tests were recorded.

\section{Assessment}

Hypertension was defined as systolic blood pressure $\geq 140$ $\mathrm{mmHg}$ or diastolic blood pressure $\geq 90 \mathrm{mmHg}$ and/or use of antihypertensive drugs according to 2013 ESC/ESH Guidelines for the management of arterial hypertension. ${ }^{18}$ Hyperlipidemia was defined as serum total cholesterol level $\geq 220 \mathrm{mg} / \mathrm{dL}$ or low-density lipoprotein cholesterol level $\geq 140 \mathrm{mg} / \mathrm{dl}$ and/or the use of lipid-lowering drugs according to $2016 \mathrm{ESC} / \mathrm{EAS}$ Guidelines for the Management of Dyslipidaemias. ${ }^{19}$ Diabetes is defined as the use of insulin or hypoglycemic agents and/or non-fasting blood glucose level $\geq 200 \mathrm{mg} / \mathrm{dl}$, and/or fasting blood glucose level $\geq 126 \mathrm{mg} / \mathrm{dl}$ according to Standards of Medical Care in Diabetes 2014. ${ }^{20}$ Smoking status was defined as never smoking (never smoking or less than 100 cigarettes in a lifetime), current smokers (still smokers or patients who quit smoking for less than half a year), and former smokers (who quit smoking at least six months before the start of the study). Smoking was defined as smoking at least one cigarette a day or at least seven cigarettes a week for half a year. ${ }^{21}$

\section{Preoperative Evaluation of Plaque Characteristics CTA Examination}

CTA was performed with 64 slice CT scanner (Somatom Sensation 64, Siemens, Forchheim, Germany). The patients were divided into calcified plaque group and noncalcified plaque group according to the difference of 
responsible plaque image and $\mathrm{HU}$ value (the area above $130 \mathrm{HU}$ in plain scan image is calcified plaque, while $<50$ $\mathrm{Hu}$ is non-calcified plaque). ${ }^{22}$

\section{Ultrasonic Examination}

Philips EPIQ7 Doppler ultrasound (Philips EPIQ7, Bothell Everett Highway, Washington, USA) with probe frequency set as 3.0-9.0 MHz, 2.0-5.0 MHz, and Hitachi Preirus (Hitachi, Tokyo, Japan) with the probe frequency set as 4.0 8.0 MHz were used. The patient's head was slightly tilted back to fully expose the neck. The head was slightly inclined to the side opposite to the side under examination. The responsible plaque was examined. High-resolution dynamic ultrasound images of all patients were saved, including transverse and longitudinal scanning. The characteristics of calcified plaque were evaluated by dynamic image playback, and the proportion of calcification in plaque was measured.

\section{A}

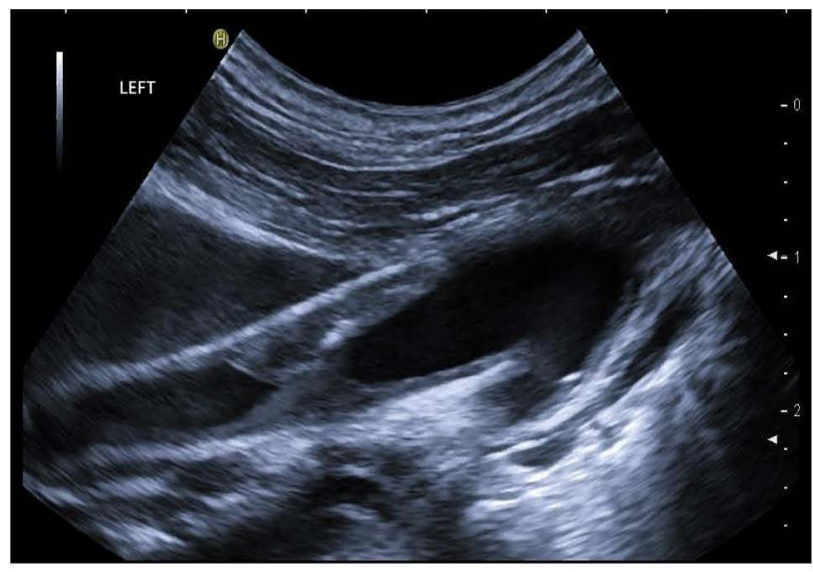

C

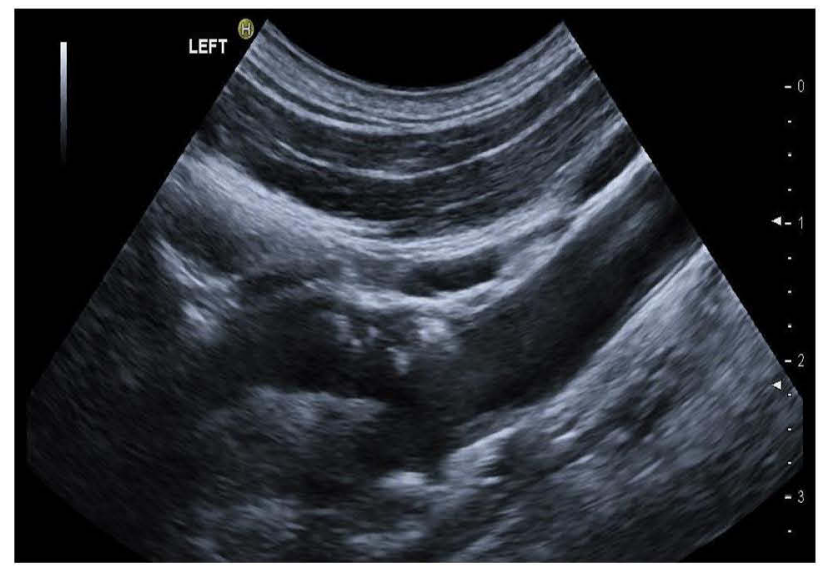

\section{Calcification Evaluation}

The characteristics of calcification include the location and size of calcification. According to the location of calcification in the plaque, the plaque can be divided into four types: basal type, top type, internal type and multi-position type (including more than two positions, Figure 1). According to the size of calcification, calcification types can be divided into three types: patchy calcification (diameter greater than $3 \mathrm{~mm}$ ), granular calcification (diameter less than $3 \mathrm{~mm}$ ); mixed type (including two types) (Figure 1). The proportion of calcification in the plaque was measured by the largest longitudinal section of the responsible plaque to measure the percentage of calcification in the plaque area. The diameter and area of calcification were measured by Unisight 4.2 software (DJ health union systems, Shanghai, China).

\section{B}

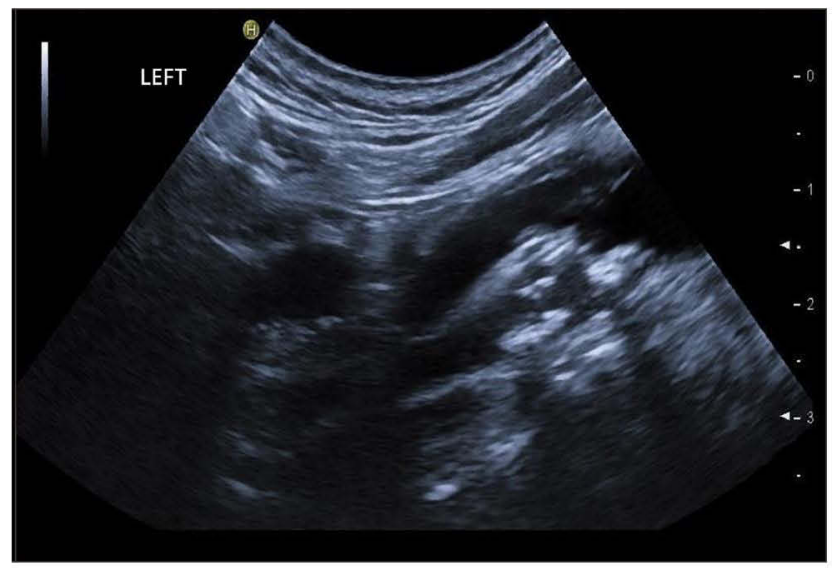

D

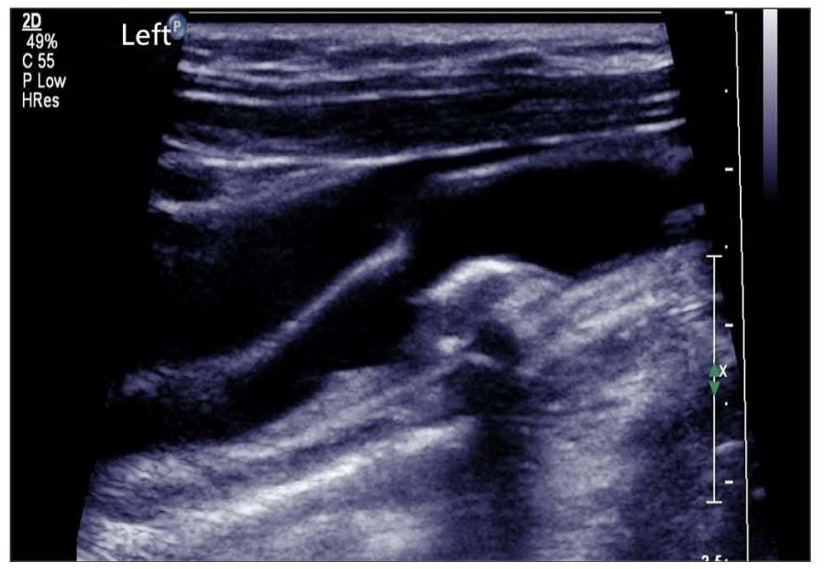

Figure I Calcification characteristics. (A) Plaque internal patchy calcification; (B) Patchy calcification in the base, internal and top of plaque; (C) Patchy calcification in base, internal of plaque; (D) Patchy calcification in the top of plaque and granular calcification in the base of plaque. 


\section{Quality Control of Plaque assessment}

The consistency of CTA and ultrasound in the evaluation of responsible plaque for calcified plaque was evaluated. $\mathrm{CT}$ and ultrasound images were evaluated by four experienced physicians, each of whom evaluated at least 2000 carotid plaque images per year. The doctors were divided into two groups, and the consistency test of reliability between groups was conducted.

\section{Surgical and Pathological Examination of Plaque \\ Surgery}

All operations were performed under general anesthesia, and TCD was used to continuously monitor the changes of cerebral blood flow during the operation. Carotid sheath was exposure, the common carotid artery external carotid artery and internal carotid artery were separated. The wall of common carotid artery and internal carotid artery was cut longitudinally, and the responsible plaque of carotid artery was dissected carefully to keep the integrity of plaque as much as possible. If the blood circulation is unobstructed, the internal carotid artery was clamped. If there is thrombosis, balloon catheter was used to remove the thrombus. Remove the attached plaque tissue carefully until the vascular wall is smooth, trim the distal intima neatly, and suture the vascular wall continuously under the operating microscope. After suturing, the blocking forceps on external carotid artery, common carotid artery and internal carotid artery were opened successively. If TCD monitoring showed that the flow of middle cerebral artery increased by $150 \%$ after opening, the common carotid artery was partially closed and then gradually opened to prevent cerebral blood perfusion. After TCD monitoring confirmed that the blood flow of the middle cerebral artery on the operative side returned to normal, the carotid artery incision was sutured in sequence.

\section{Pathological Evaluation of Plaque After Carotid Endarterectomy}

After the operation, the plaques were fixed in neutral formalin solution, then decalcified with EDTA solution, dehydrated routinely, and embedded in paraffin. Slices with thickness of $4 \mu \mathrm{m}$ were obtained at the interval of $1 \mathrm{~mm}$, and then stained with HE. Two experienced pathologists evaluated and recorded IPH and LRNC. The two pathologists were not aware of the imaging results of the patients, and they evaluated results with double-blind method.

\section{Statistical Analysis}

Continuous variables are expressed as mean $\pm \mathrm{SD}$, while categorical variables are expressed as percentages. Paired $t$-test or ANOVA was used for comparison of continuous variable groups, and chi-square test was used for comparison of categorical variable groups. Multivariate logistic regression model was used to evaluate the relationship between calcification and major risk factors. Kappa test was used to test the consistency of two imaging and physician assessment of plaque calcification. The normal distribution of continuous variable distribution was evaluated by Shapiro Wilk test. All statistical analyses were performed using IBM SPSS statistics 23.0 software (International Business Machines Corporation, Armonk, New York, N.Y., USA). P $<0.05$ was considered as statistically significant difference.

\section{Results \\ Basic Characteristics}

A total of 142 patients were included in the study. There were 119 males $(83.8 \%)$ and 23 females (16.2\%), aged $38-82$ years (mean age $63.6 \pm 7.2$ years). There were 96 cases $(67.6 \%)$ with hypertension, 45 cases $(31.7 \%)$ with diabetes and 43 cases $(30.3 \%)$ with hyperlipidemia. There were 55 non-smokers (38.7\%), 56 smokers (39.4\%) and 31 former smokers $(21.8 \%)$.

Ultrasonography and CTA were used to evaluate the calcification of responsible plaque in 142 cases, including 119 (83.8) calcified plaque and 23 (16.2) non-calcified plaque. The calcification characteristics of 119 cases of calcified plaque were as follows: basal calcification in 49 cases $(41.2 \%)$, internal calcification in 19 cases $(16.0 \%)$, top calcification in 14 cases $(11.8 \%)$, multi-location calcification in 37 cases $(31.1 \%)$; patchy calcification in 45 cases $(37.8 \%)$, granular calcification in 29 cases $(22.7 \%)$, and mixed type calcification in 45 cases $(37.8 \%)$.

There was a good consistency between groups and within groups in the evaluation of calcification. The consistency between groups was, calcification $(\mathrm{k}=0.96)$, calcification location $(\mathrm{k}=0.92)$ and calcification type $(\mathrm{k}=$ $0.90)$. The intra-group consistency was as follows, calcified plaque $(\mathrm{k}=0.94)$, calcification location $(\mathrm{k}=0.89)$ and calcification type $(\mathrm{k}=0.88)$.

\section{Correlation Between LRNC, IPH and Clinical Risk Factors}

Of the 142 plaque specimens, 78 (54.9\%) had LRNC and 41 $(28.9 \%)$ had IPH (Table 1). It was found that plaque with 
Table I Comparison of Clinical Risk Factors Between Patients with LRNC and IHP and without LRNC and IHP

\begin{tabular}{|c|c|c|c|c|c|c|}
\hline & $\begin{array}{l}\text { With LRNC } \\
(n=78)\end{array}$ & $\begin{array}{l}\text { Without LRNC } \\
(n=64)\end{array}$ & $\mathbf{P}$ & $\begin{array}{l}\text { With IPH } \\
(n=4 I)\end{array}$ & $\begin{array}{l}\text { Without IPH } \\
(n=101)\end{array}$ & $\mathbf{P}$ \\
\hline Gender (male) & $68(87.2)$ & $5 I(79.7)$ & 0.228 & $34(82.9)$ & $85(84.2)$ & 0.857 \\
\hline Age (years) & $65.4 \pm 7.1$ & $61.5 \pm 6.9$ & $0.001 *$ & $64.0 \pm 6.2$ & $63.5 \pm 7.6$ & 0.726 \\
\hline BMI $\left(\mathrm{kg} / \mathrm{m}^{2}\right)$ & $24.9 \pm 2.6$ & $25.2 \pm 2.9$ & 0.635 & $24.6 \pm 2.9$ & $25.2 \pm 2.6$ & 0.245 \\
\hline Smoking history & $31 / 31 / 16$ & $24 / 25 / 15$ & 0.910 & $31 / 31 / 16$ & $24 / 25 / 15$ & 0.910 \\
\hline Smoking, pack-year & $21.1 \pm 24.9$ & $16.8 \pm 20.6$ & 0.265 & $15.6 \pm 20.0$ & $20.6 \pm 24.1$ & 0.243 \\
\hline Hypertension & $56(71.8)$ & $40(62.5)$ & 0.239 & $56(71.8)$ & $40(62.5)$ & 0.239 \\
\hline Diabetes & $25(32.1)$ & $20(31.3)$ & 0.919 & $25(32.1)$ & $20(31.3)$ & 0.919 \\
\hline Hyperlipidemia & $25(32.1)$ & $18(28.1)$ & 0.612 & $25(32.1)$ & $18(28.1)$ & 0.612 \\
\hline $\begin{array}{l}\text { Fasting blood glucose } \\
(\mathrm{mmol} / \mathrm{L})\end{array}$ & $6.0 \pm 2.0$ & $5.6 \pm 1.7$ & 0.219 & $5.5 \pm 1.5$ & $5.9 \pm 2.0$ & 0.326 \\
\hline Triglyceride (mmol/L) & $1.6 \pm 1.4$ & $1.6 \pm 0.9$ & 0.981 & $1.9 \pm 1.8$ & $1.5 \pm 0.8$ & 0.123 \\
\hline Cholesterol (mmol/L) & $3.3 \pm 1.0$ & $3.5 \pm 0.9$ & 0.370 & $3.5 \pm 0.9$ & $3.3 \pm 1.0$ & 0.342 \\
\hline $\begin{array}{l}\text { High density lipoprotein } \\
(\mathrm{mmol} / \mathrm{L})\end{array}$ & $1.3 \pm 0.4$ & $1.3 \pm 0.4$ & 0.563 & $1.2 \pm 0.3$ & $1.3 \pm 0.4$ & 0.200 \\
\hline $\begin{array}{l}\text { Low density lipoprotein } \\
(\mathrm{mmol} / \mathrm{L})\end{array}$ & $2.0 \pm 0.8$ & $2.1 \pm 0.7$ & 0.404 & $2.1 \pm 0.7$ & $2.0 \pm 0.8$ & 0.618 \\
\hline
\end{tabular}

Note: $* \mathrm{P}<0.05$ was considered as statistically significant difference.

LRNC was more common in older people $(65.4 \pm 7.1$ years vs $61.5 \pm 6.9$ years; $\mathrm{P}=0.001)$, but other risk factors were not significantly different from those without LRNC (all P > 0.05). There was no significant difference in clinical risk factors between IHP group and non-IHP group (all P > 0.05).

\section{Correlation Between LRNC and Plaque Calcification}

93.6\% of the 78 plaques in LRNC group had calcification, while it was $71.9 \%$ in non-LRNC group $(\mathrm{P}=0.000)$, indicating that $\mathrm{LRNC}$ was more likely to appear in calcified plaques (Table 2). LRNC was often found in multiple calcification ( $\mathrm{P}$ $=0.003)$ and mixed type calcification $(\mathrm{P}=0.001)$ (Figure 2$)$. However, there was no significant difference in the presence or absence of LRNC in other single position calcified plaques and single size of calcified plaques $(\mathrm{P}>0.05)$ (Table 2).

In univariate regression analysis, it was found that the presence or absence of intraplaque calcification (OR, 5.71; 95\% CI, 2.0-16.44; $\mathrm{P}=0.001$ ), multiple intra plaque calcification (OR, 3.82; 95\% CI, 1.65-8.84; $\mathrm{P}=0.002)$ and mixed types of calcification (OR, 3.72; 95\% CI, 1.69-8.19;
$\mathrm{P}=0.001)$ were significantly associated with LRNC, but not with other calcification features (all $\mathrm{P}<0.05$ ) (Table 3).

In order to exclude the influence of other interference factors, we included the characteristics of plaque calcification and other factors that may affect LRNC into the multiple factor regression equation. The results showed that LRNC was still significantly associated with the presence of intraplaque, multiple intra plaque calcification and mixed types of calcification (all $\mathrm{P}<0.05$ ). Multilocation calcification and mixed type calcification in plaque were independent risk factors of LRNC.

\section{Correlation Between IPH and Plaque Calcification}

The incidence of calcification in IPH group was lower than that in LRNC group (87.8\%), and there was no significant difference between IPH group and non-IPH group ( $\mathrm{P}=$ 0.410). However, in the comparison of calcification sites between the two groups, it was found that multiple calcification was also easy to merge with IPH $(\mathrm{P}=0.008)$ (Figure 3$)$, which was similar with LRNC group. In contrast, basal calcification was not associated with IPH $(\mathrm{P}=0.002)$. 
Table 2 Comparison of Calcification Characteristics Between LRNC Group and Non LRNC Group

\begin{tabular}{|l|l|l|l|}
\hline & With LRNC (n=78) & Without LRNC (n=64) & P \\
\hline Calcification (\%) & $73(93.6)$ & $46(71.9)$ & $0.000^{*}$ \\
\hline Amount of calcification (\%) & $13.7 \pm 16.2$ & $18.0 \pm 17.3$ & 0.132 \\
\hline Calcification location & & & \\
Basal calcification (\%) & $22(28.2)$ & $27(42.2)$ & 0.08 \\
Internal calcification (\%) & $14(17.9)$ & $5(7.8)$ & 0.078 \\
Top calcification (\%) & $9(11.5)$ & $5(7.8)$ & 0.459 \\
Multiple calcification (\%) & $28(35.9)$ & $9(14.1)$ & $0.003^{*}$ \\
\hline Size of calcification & & & \\
Patchy calcification (\%) & $23(29.5)$ & $22(34.4)$ & $13(20.3)$ \\
Granular calcification (\%) & $16(20.5)$ & $11(17.2)$ & 0.533 \\
Mixed type calcification (\%) & $34(43.6)$ & 0.976 \\
\hline
\end{tabular}

Note: $* \mathrm{P}<0.05$ was considered as statistically significant difference.

In the comparison of calcification types, smaller granular calcification was easier to merge with IPH, while the larger patchy calcification and mixed type calcification were not associated with IPH $(\mathrm{P}>0.05)$ (Table 4).
In univariate regression analysis of IPH and calcification characteristics, simple basal calcification was still a protective factor for IPH (OR, 0.23 ; $95 \%$ CI, 0.09 $0.60 ; \mathrm{P}=0.003)$, while multiple location calcification

A

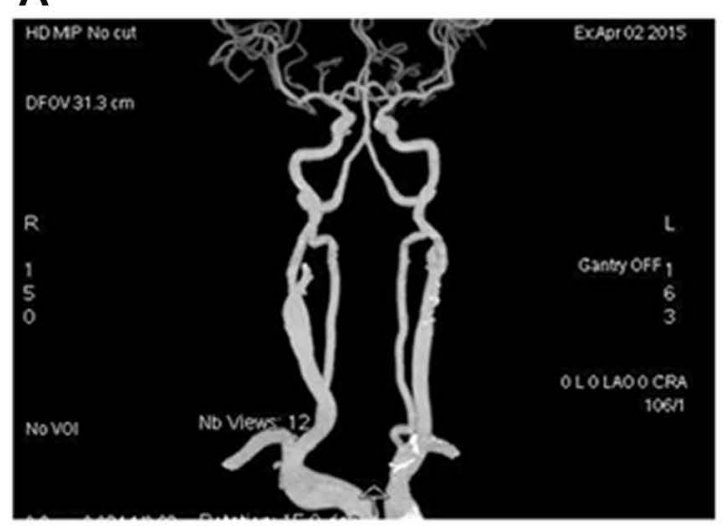

C

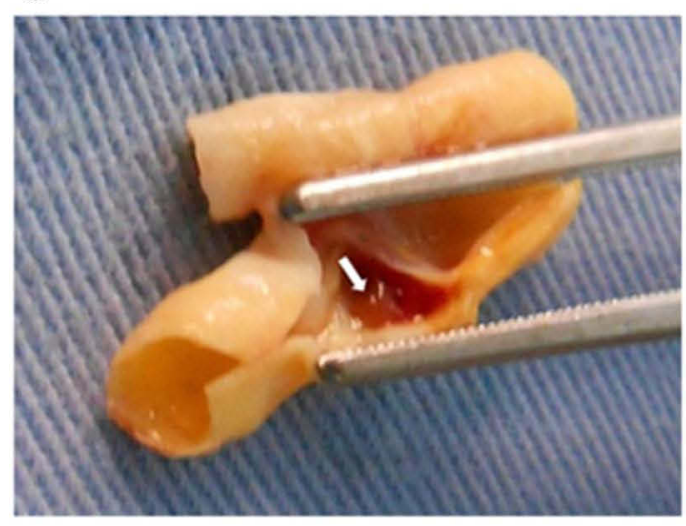

B

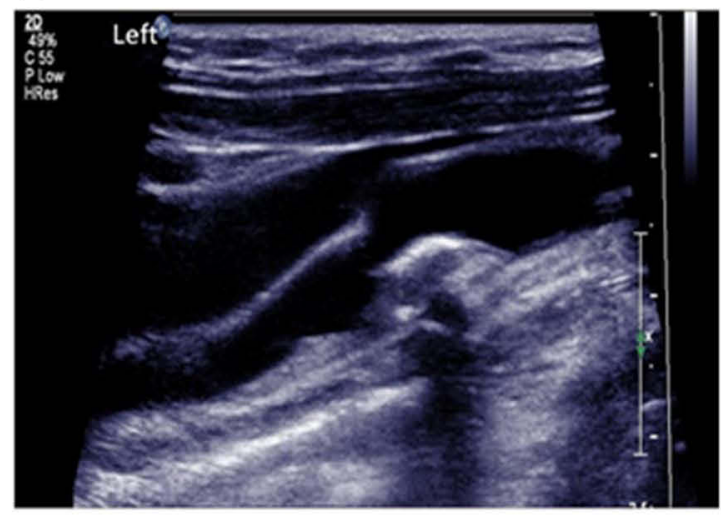

D

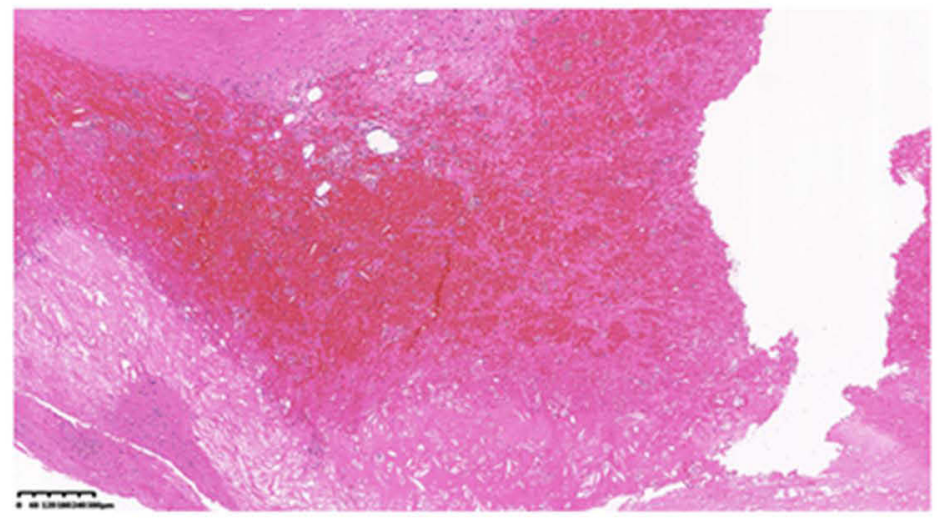

Figure 2 A 66 years old female patient with severe stenosis near the bifurcation of left carotid artery before and after CEA. (A) CTA showed calcification in the left responsible plaque; (B) High resolution ultrasound showed calcification at the top and the base, with calcification diameter $>3$ mm, which was classified as multi location, patchy calcified plaque; (C) Intraoperative hemorrhage was found in the plaque (white arrow); (D) A large number of red blood cells were seen on the pathological section. 
Table 3 Regression Analysis of Plaque Calcification Characteristics and LRNC

\begin{tabular}{|l|l|l|l|l|l|l|}
\hline \multirow{2}{*}{} & \multicolumn{2}{l|}{ Single Factor Regression Analysis } & \multicolumn{2}{l|}{ Multivariate Regression Analysis } \\
\cline { 2 - 7 } & OR & Cl (95\%) & P & OR & CI (95\%) & P \\
\hline Calcification & 5.71 & $2.0-16.44$ & 0.001 & 9.02 & $2.63-30.96$ & $0.000^{*}$ \\
Basal calcification & 0.47 & $0.23-0.96$ & 0.039 & 0.51 & $0.23-1.13$ & 0.097 \\
Internal calcification & 2.58 & $0.88-7.60$ & 0.085 & 2.63 & $0.8-8.63$ & 0.110 \\
Top calcification & 1.54 & $0.49-4.85$ & 0.461 & 2.01 & $0.58-6.90$ & 0.269 \\
Multiple calcification & 3.82 & $1.65-8.84$ & 0.002 & 3.78 & $1.51-9.5$ & $0.005^{*}$ \\
Patchy calcification & 0.80 & $0.39-1.62$ & 0.534 & 0.93 & $0.42-2.04$ & 0.846 \\
Granular calcification & 1.01 & $0.45-2.3$ & 0.976 & 1.21 & $0.49-3.0$ & 0.684 \\
Mixed type calcification & 3.72 & $1.69-8.19$ & 0.001 & 3.37 & $1.44-7.88$ & $0.005^{*}$ \\
\hline
\end{tabular}

Notes: Adjusted for age, gender, diabetes, hypertension, hyperlipidemia, smoking, ${ }^{*} \mathrm{P}<0.05$ was considered as statistically significant difference.

(OR, 2.87; 95\% CI, 1.30-6.33; P = 0.009) and granular calcification (OR, 2.47; 95\% CI, 1.06-5.76; $\mathrm{P}=0.037$ ) were associated with the occurrence of IPH (Table 5). However, in the multivariate regression analysis, after adjusting other influencing factors, granular calcification was not significantly correlated with IPH $(\mathrm{P}=0.058)$, indicating that the location of calcification may be related to the occurrence of IPH.
A

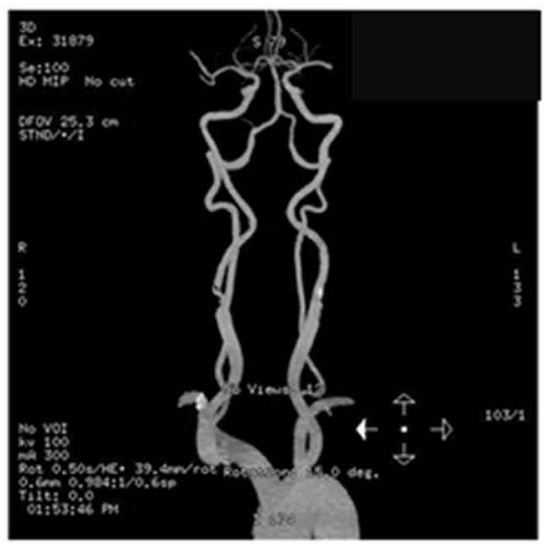

C

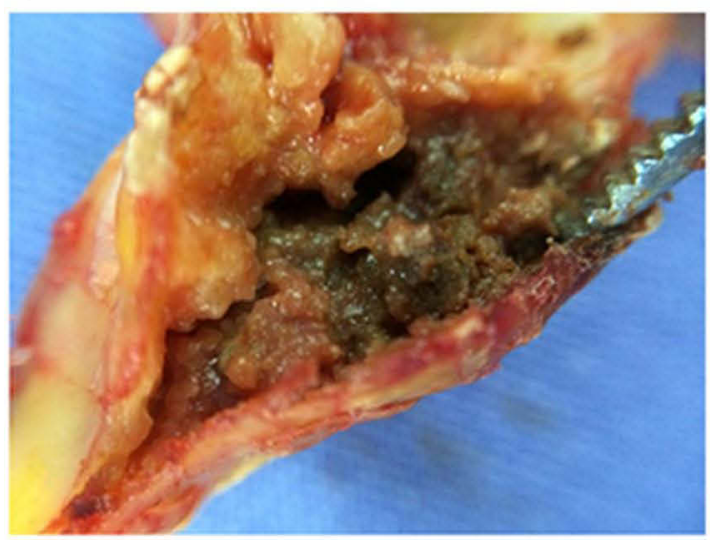

B

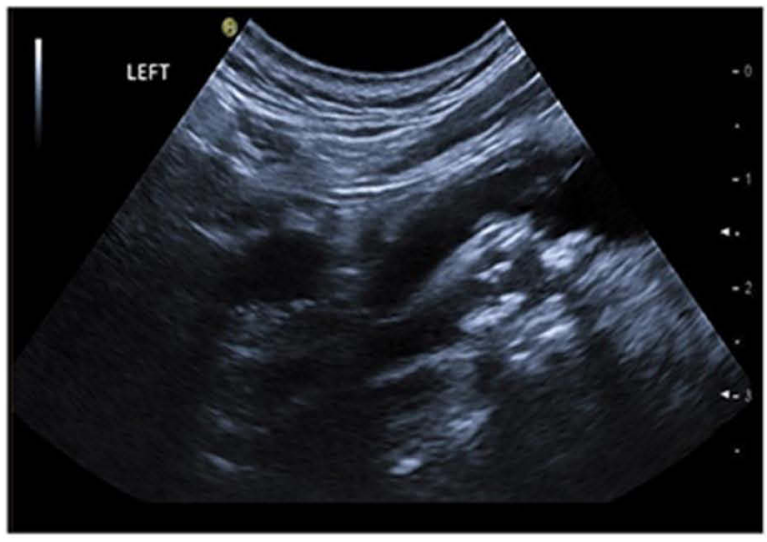

D

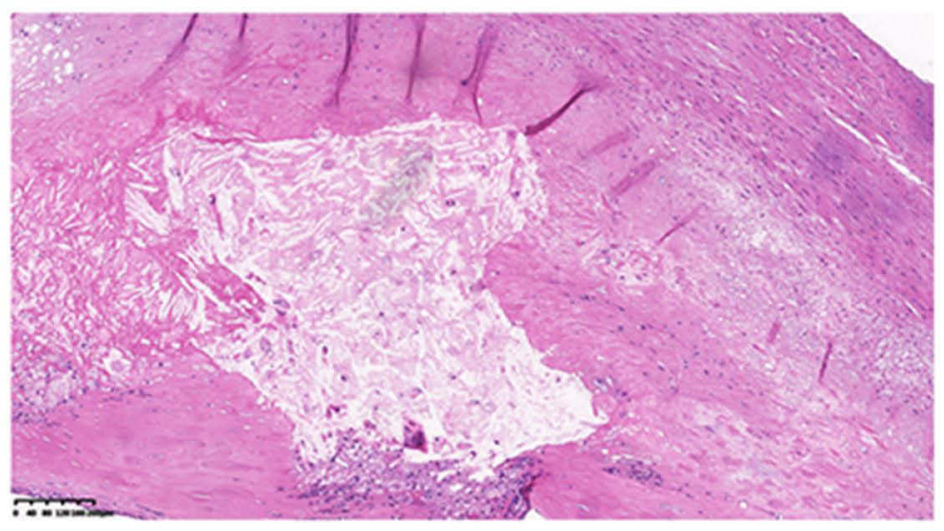

Figure 3 A 70 years old male patient with severe stenosis of the carotid bulb of the left superficial femoral artery before and after CEA. (A) CTA showed calcified plaque in the left bulb; (B) High resolution ultrasound showed that there were multiple calcification sites and different sizes, which were classified as multi location and mixed calcification plaque; (C) During the operation, a large number of lipid dead bodies were found in the plaque; (D) The pathological section showed lipid accumulation (cholesterol crystal) accompanied by degeneration of fiber and collagen and inflammatory cells. 
Table 4 Comparison of Calcification Characteristics Between Patients with and without IPH

\begin{tabular}{|l|l|l|l|}
\hline & With IPH (n=4I) & Without IPH (n= I0I) & P \\
\hline Calcification (\%) & $36(87.8)$ & $83(82.2)$ & 0.410 \\
Amount of calcification & $15.1 \pm 17.1$ & $16.4 \pm 16.8$ & 0.676 \\
Basal calcification (\%) & $6(14.6)$ & $43(42.6)$ & $0.002^{*}$ \\
Internal calcification (\%) & $7(17.1)$ & $12(11.9)$ & 0.410 \\
Top calcification (\%) & $8(14.6)$ & $6(7.9)$ & 0.224 \\
Multiple calcification (\%) & $17(41.5)$ & $20(19.8)$ & $0.008^{*}$ \\
Patchy calcification (\%) & $9(22.0)$ & $36(35.6)$ & 0.112 \\
Granular calcification (\%) & $13(31.7)$ & $16(15.8)$ & $0.034^{*}$ \\
Mixed type calcification (\%) & $14(34.1)$ & $31(30.7)$ & 0.689 \\
\hline
\end{tabular}

Note: $* \mathrm{P}<0.05$ was considered as statistically significant difference.

Table 5 Regression Analysis of Plaque Calcification Characteristics and IPH

\begin{tabular}{|l|l|l|l|l|l|l|}
\hline & \multicolumn{2}{l|}{ Single Factor Regression Analysis } & \multicolumn{2}{l|}{ Multivariate Regression Analysis } \\
\cline { 2 - 7 } & OR & Cl (95\%) & P & OR & Cl (95\%) & P \\
\hline Calcification & 1.56 & $0.54-4.53$ & 0.412 & 1.87 & $0.61-5.73$ & 0.271 \\
Basal calcification & 0.23 & $0.09-0.60$ & 0.003 & 0.25 & $0.09-0.66$ & $0.005^{*}$ \\
Internal calcification & 1.53 & $0.56-4.2$ & 0.413 & 1.25 & $0.42-3.71$ & 0.685 \\
Top calcification & 1.99 & $0.65-6.15$ & 0.231 & 2.03 & $0.62-6.69$ & 0.244 \\
Multiple calcification & 2.87 & $1.30-6.33$ & 0.009 & 3.58 & $1.49-8.61$ & $0.004^{*}$ \\
Patchy calcification & 0.51 & $0.22-1.18$ & 0.116 & 0.50 & $0.20-1.24$ & 0.135 \\
Granular calcification & 2.47 & $1.06-5.76$ & 0.037 & 2.39 & $0.97-5.86$ & 0.058 \\
Mixed type calcification & 1.17 & $0.54-2.53$ & 0.689 & 1.33 & $0.57-3.09$ & 0.506 \\
\hline
\end{tabular}

Notes: Adjusted for age, gender, diabetes, hypertension, hyperlipidemia, smoking, ${ }^{*} \mathrm{P}<0.05$ was considered as statistically significant difference.

\section{Discussion}

In current study, we found that the calcification characteristics in carotid plaques were closely related to LRNC and IPH. The pathological features of LRNC are accumulation of cell debris and lipid in necrotic cells, accompanied by a large number of macrophage infiltration and inflammatory reaction, which is a marker change of vulnerable plaque. $^{23}$ The formation and expansion of LRNC play a key role in the pathology of unstable atherosclerotic plaque. ${ }^{24}$ In this study, we found that the presence of plaque calcification is closely related to LRNC, especially multi-location calcification and mixed type calcification. The amount of calcification in plaque and single location or single type of calcification were not significant correlated with LRNC. Some histological evidence might explain the association between calcification in plaques and LRNC. It was found that the increase of intracellular calcium level could induce plaque cell necrosis. ${ }^{25}$ Once plaque cells were necrotic, they released their cytoplasm into large lipid masses. At the same time, it was reported that a large number of calcification could be detected in the lipid core. ${ }^{26}$ Proudfoot et $\mathrm{al}^{27}$ considered that calcification was the terminal state of tissue in the pathological process of atherosclerosis, while necrosis was the end point. In addition, macrophages and inflammatory changes in LRNC may be the relational factors between calcification and necrosis. Ikeda et al showed that macrophages could induce osteogenic expression in vascular smooth muscle cells and promote the formation of calcification in vascular wall. ${ }^{28,29}$ Other studies have also showed that atherosclerotic calcification is driven by apoptotic inflammatory cells and is a powerful indicator of chronic inflammatory diseases. ${ }^{30,31}$

Our results showed that multiple calcification and mixed calcification were closely related to the presence of LRNC in plaques classified according to the location and size of calcification. These two calcification characteristics reveal the complex distribution and size heterogeneity of calcification in the plaque. However, the mechanism of this phenomenon is not clear. We speculate that these two calcification characteristics increase the internal complexity of the plaque and change the stress in the plaque 
compared with single location and single type of calcification. Studies have shown that the hardness of calcification in plaques is at least 4-5 times higher than that of cellular components, ${ }^{32}$ and its internal stress tends to concentrate on the interface between calcified and non-calcified areas. ${ }^{33}$ The complex distribution and uneven size of calcification increase the interface between the soft and hard tissues in the plaque, resulting in the local concentration of unstable force in the plaque and the asymmetry in the plaque, which may contribute to the progress of inflammation and the formation of lipid core. ${ }^{34}$

The pathogenesis of IPH is still unclear. Most researchers believe that immature neovascularization rupture may be the pathological basis. Under the effect of inflammation and hypoxia, immature neovascularization gradually increases and invades into the plaque from the adventitia, which is called intraplaque neovascularization. After the rupture of neovascularization due to various reasons, hemorrhage in the plaque results in rapid increase of plaque volume and obvious increase of instability. ${ }^{35,36}$ Takaya et $\mathrm{al}^{37}$ have shown that the risk of stroke is greatly increased by more than 5 times due to intra plaque hemorrhage. Our study found that multiple location calcification was closely related to IPH, which was consistent with previous studies. ${ }^{14,38}$ The mechanism of IPH may be similar to that of LRNC. It is speculated that biomechanical changes are the important reason. Compared with the soft surrounding components, such as neovascularization, calcification has higher hardness and less possibility of deformation. Therefore, there may be destructive stress at the interface between calcification and soft components, which may lead to the rupture of new blood vessels. Compared with single-site calcification, if there are multiple position calcifications in the plaque, the interface of different density tissues will increase, and the tissue between them will bear higher mechanical load, ${ }^{16,34}$ resulting in the formation and distortion of new blood vessels, thus increasing the possibility of new blood vessel rupture. Our results were consistent with a previous study, which pointed out that local stress and tensile changes in plaque tended to appear around vulnerable new vessels in carotid plaques, that might lead to the deformation of the region, which leaded to the occurrence of IPH due to vascular rupture. ${ }^{39}$ Unlike the results of multi-location calcification, we found that simple basal calcification could reduce the occurrence of intra plaque hemorrhage. On one hand, basal calcification is far away from the lumen and has little effect on plaque stress. On the other hand, the base of the plaque is close to the intima, which may hinder the growth of neovascularization from adventitia to intima, thus preventing the formation of IPH. For the size of calcification, this study found that small granular calcification in the plaque was correlated with IPH. It was speculated that the smaller calcification was related to more angulation of surrounding tissues. However, its OR value was relatively low. After adjusting for other clinical risk factors, this correlation had no statistical significance.

Nandalur et $\mathrm{al}^{40}$ measured the calcified components and non-calcified components of plaque by CTA, and the results showed that the proportion of calcified components rather than calcified volume in patients with carotid artery stenosis was correlated with plaque stability. When calcification accounted for more than $45 \%$ of the total volume, and the occurrence of clinical symptoms was significantly reduced. However, another study emphasized that the more calcified plaques were associated with IPH, while LRNC was less common, which indicated that the larger amount of calcification in the plaque did not mean that it was more stable. ${ }^{13}$ In our study, we found that the proportion of calcification in plaques was not significantly correlated with LRNC and IPH, but only related to specific calcification characteristics. Although the conclusions of previous studies were different from our results, we concluded that the assessment of plaque vulnerability could be based on the characteristics of calcification, while the location and type of calcification were related to the plaque vulnerability, which was more important. ${ }^{9,16}$

In current study, we observed the relationship between gender, age, body mass index, hypertension, hyperglycemia, hyperlipidemia, smoking and LRNC, IPH. It was found that the older population was closely related to the occurrence of LRNC, which was consistent with the previous study. Gennaro et $\mathrm{al}^{41}$ showed that with the growth of age, the normal apoptosis mechanism of vascular smooth muscle cells would be damaged, and the process of apoptosis would be accompanied by the formation of large necrotic core in plaque. ${ }^{42}$ We found that there was no significant correlation between other risk factors and the occurrence of LRNC and IPH. A previous study showed that hypertension, and male were independent risk factors for IPH. ${ }^{43}$ In order to exclude the influence of these potential risk factors, we included them together with calcification characteristics in multivariate regression analysis. The results showed that the correlation between calcification characteristics and LRNC and IPH still existed. 
Many studies have also shown that the intraplaque IPH and LRNC are important predictors of plaque vulnerability. The effect of other plaque components, especially calcification, on plaque vulnerability is still controversial. More detailed observation of calcification features, such as calcification location, calcification type or calcification volume, etc., which will play a more important role in promoting the assessment of plaque vulnerability. In current study, we found that the calcification characteristics in carotid plaques were closely related to LRNC and IPH. Multiple calcification and mixed calcification were closely related to the presence of LRNC in plaques classified according to the location and size of calcification. Moreover, we observed the relationship between gender, age, body mass index, hypertension, hyperglycemia, hyperlipidemia, smoking and LRNC, IPH.

There are still some limitations in this study. First, this is a retrospective study. There was no analysis of clinical symptoms and medication. Second, we use the proportion of calcification in the largest longitudinal section of the plaque to estimate the proportion of calcification in the whole plaque. There may be some deviation, which may lead to the deviation of the results. Multisection function of three-dimensional volume probe should be used in further study. In addition, the patients included in this study were all patients with severe stenosis, and some cases might have plaque ulcer and plaque surface thrombosis, which were also important characteristics of vulnerable plaque. We did not conduct detailed subgroup analysis, which might have an impact on the evaluation of the results, which needed to be further investigated after refinement. The history of stroke/TIA of the patients undergoing CAE and the history of myocardial infarction of the patients were not recorded and analyzed in this study. Other important morphological features, such as thrombus, fibrin, inflammatory infiltration have not been evaluated in this study.

\section{Conclusions}

In conclusion, calcification characteristics of carotid atherosclerotic plaques are closely related to the vulnerability of plaques, especially multi-location calcification and mixed type calcification. This is of great significance for the detection of vulnerable plaques and may provide new insights into the pathogenesis of LRNC and IPH.

\section{Abbreviations}

LRNC, lipid rich necrotic core; IPH, intraplaque hemorrhage; CEA, carotid endarterectomy; CTA, Computed tomographic angiography; VP, vulnerable plaques; CDU, color Doppler ultrasound; DSA, digital subtraction angiography; BMI, body mass index.

\section{Data Sharing Statement}

The datasets used or analysed during the current study are available from the corresponding author on reasonable request.

\section{Ethics Approval and Informed Consent}

All procedures performed in studies involving human participants were in accordance with the ethical standards of the institutional and/or national research committee and with the 1964 Helsinki declaration and its later amendments or comparable ethical standards. This study is approved by relevant Ethics Committee of the Xuanwu Hospital, Capital Medical University, Beijing, China. All patients gave informed written consent to participate in the study.

\section{Consent for Publication}

Informed consent was obtained from all individual participants included in the study.

\section{Author Contributions}

All authors made a significant contribution to the work reported, whether that is in the conception, study design, execution, acquisition of data, analysis and interpretation, or in all these areas; took part in drafting, revising or critically reviewing the article; gave final approval of the version to be published; have agreed on the journal to which the article has been submitted; and agree to be accountable for all aspects of the work.

\section{Funding}

There is no funding to report.

\section{Disclosure}

The authors declare that they have no conflicts of interest.

\section{References}

1. Mohammed N, Anand SS. Prevention of disabling and fatal strokes by successful carotid endarterectomy in patients without recent neurological symptoms: randomized controlled trial. MRC asymptomatic carotid surgery trial (ACST) collaborative group. Vasc Med. 2005;10 (1):77-78. doi:10.1191/1358863x05vm588xx 
2. Albers GW, Amarenco P, Easton JD, Sacco RL, Teal P. Antithrombotic and thrombolytic therapy for ischemic stroke: the Seventh ACCP Conference on antithrombotic and thrombolytic therapy. Chest. 2004;126(3 Suppl):483s-512s. doi:10.1378/chest.126.3_suppl.483S

3. Serfaty JM, Nonent M, Nighoghossian N, et al. Plaque density on CT, a potential marker of ischemic stroke. Neurology. 2006;66 (1):118-120. doi:10.1212/01.wnl.0000191391.71614.51

4. Gao T, Zhang Z, Yu W, Zhang Z, Wang Y. Atherosclerotic carotid vulnerable plaque and subsequent stroke: a high-resolution MRI study. Cerebrovasc Dis. 2009;27(4):345-352. doi:10.1159/000202011

5. Redgrave JN, Lovett JK, Rothwell PM. Histological features of symptomatic carotid plaques in relation to age and smoking: the oxford plaque study. Stroke. 2010;41(10):2288-2294. doi:10.1161/ STROKEAHA.110.587006

6. Spagnoli LG, Mauriello A, Sangiorgi G, et al. Extracranial thrombotically active carotid plaque as a risk factor for ischemic stroke. JAMA. 2004;292(15):1845-1852. doi:10.1001/jama.292.15.1845

7. Wahlgren CM, Zheng W, Shaalan W, Tang J, Bassiouny HS. Human carotid plaque calcification and vulnerability. Relationship between degree of plaque calcification, fibrous cap inflammatory gene expression and symptomatology. Cerebrovasc Dis. 2009;27(2):193-200. doi:10.1159/000189204

8. Alsheikh-Ali AA, Kitsios GD, Balk EM, Lau J, Ip S. The vulnerable atherosclerotic plaque: scope of the literature. Ann Intern Med. 2010;153 (6):387-395. doi:10.7326/0003-4819-153-6-201009210-00272

9. Finn AV, Nakano M, Narula J, Kolodgie FD, Virmani R. Concept of vulnerable/unstable plaque. Arterioscler Thromb Vasc Biol. 2010;30 (7):1282-1292. doi:10.1161/ATVBAHA.108.179739

10. van Gils MJ, Vukadinovic D, van Dijk AC, Dippel DW, Niessen WJ. van der Lugt A. Carotid atherosclerotic plaque progression and change in plaque composition over time: a 5-year follow-up study using serial CT angiography. AJNR Am J Neuroradiol. 2012;33 (7):1267-1273. doi:10.3174/ajnr.A2970

11. Nandalur KR, Baskurt E, Hagspiel KD, Phillips CD, Kramer CM. Calcified carotid atherosclerotic plaque is associated less with ischemic symptoms than is noncalcified plaque on MDCT. AJR Am J Roentgenol. 2005;184(1):295-298. doi:10.2214/ajr.184.1.01840295

12. Kwee RM. Systematic review on the association between calcification in carotid plaques and clinical ischemic symptoms. J Vasc Surg. 2010;51(4):1015-1025. doi:10.1016/j.jvs.2009.08.072

13. van den Bouwhuijsen QJ, Bos D, Ikram MA, et al. Coexistence of calcification, intraplaque hemorrhage and lipid core within the asymptomatic atherosclerotic carotid plaque: the Rotterdam Study. Cerebrovasc Dis. 2015;39(5-6):319-324. doi:10.1159/000381138

14. Lin R, Chen S, Liu G, Xue Y, Zhao X. Association between carotid atherosclerotic plaque calcification and intraplaque hemorrhage: a Magnetic Resonance Imaging Study. Arterioscler Thromb Vasc Biol. 2017;37(6):1228-1233. doi:10.1161/ATVBAHA.116.308360

15. Zhongzhao T, Jing H, Sadat U, et al. How does juxtaluminal calcium affect critical mechanical conditions in carotid atherosclerotic plaque? An exploratory study. IEEE Trans Biomed Eng. 2014;61 (1):35-40. doi:10.1109/TBME.2013.2275078

16. Abedin M, Tintut Y, Demer LL. Vascular calcification: mechanisms and clinical ramifications. Arterioscler Thromb Vasc Biol. 2004;24 (7):1161-1170. doi:10.1161/01.ATV.0000133194.94939.42

17. Hoshino M, Kawai H, Sarai M, et al. Noninvasive assessment of stenotic severity and plaque characteristics by coronary $\mathrm{CT}$ angiography in patients scheduled for carotid artery revascularization. $J$ Atheroscler Thromb. 2018;25(10):1022-1031. doi:10.5551/jat.42176

18. Mancia G, Fagard R, Narkiewicz K, ESH/ESC Task Force for the Management of Arterial Hypertension. 2013 Practice guidelines for the management of arterial hypertension of the European Society of Hypertension (ESH) and the European Society of Cardiology (ESC): ESH/ESC Task Force for the Management of Arterial Hypertension. $J$ Hypertens. 2013;31(10):1925-1938. doi:10.1097/HJH.0b013e32 $8364 \mathrm{ca} 4 \mathrm{c}$
19. Catapano AL, Graham I, De Backer G, et al. 2016 ESC/EAS guidelines for the management of dyslipidaemias. Eur Heart J. 2016;37 (39):2999-3058. doi:10.1093/eurheartj/ehw272

20. American Diabetes Association. Standards of medical care in diabetes--2014. Diabetes Care. 2014;37(Suppl 1):S14-80. doi:10.2337/dc14-S014

21. Krishnan A, Yadav K, Kaur M, Kumar R. Epidemiology to public health intervention for preventing cardiovascular diseases: the role of translational research. Indian J Med Res. 2010;132(5):643-650. doi:10.4103/0971-5916.73421

22. de Weert TT, Ouhlous M, Meijering E, et al. In vivo characterization and quantification of atherosclerotic carotid plaque components with multidetector computed tomography and histopathological correlation. Arterioscler Thromb Vasc Biol. 2006;26(10):2366-2372. doi:10.1161/01.ATV.0000240518.90124.57

23. Tabas I. Consequences and therapeutic implications of macrophage apoptosis in atherosclerosis: the importance of lesion stage and phagocytic efficiency. Arterioscler Thromb Vasc Biol. 2005;25 (11):2255-2264. doi:10.1161/01.ATV.0000184783.04864.9f

24. Fok PW. Growth of necrotic cores in atherosclerotic plaque. Mathematical Med Biol. 2012;29(4):301-327. doi:10.1093/imammb/ dqr012

25. Martinet W, Schrijvers DM, De Meyer GR. Necrotic cell death in atherosclerosis. Basic Res Cardiol. 2011;106(5):749-760. doi:10.1007/s00395-011-0192-x

26. Stary HC. Natural history of calcium deposits in atherosclerosis progression and regression. Z Kardiol. 2000;89(Suppl 2):28-35. doi: $10.1007 /$ s003920070097

27. Proudfoot D, Skepper JN, Hegyi L, Bennett MR, Shanahan CM, Weissberg PL. Apoptosis regulates human vascular calcification in vitro: evidence for initiation of vascular calcification by apoptotic bodies. Circ Res. 2000;87(11):1055-1062. doi:10.1161/01. RES.87.11.1055

28. Ikeda K, Souma Y, Akakabe Y, et al. Macrophages play a unique role in the plaque calcification by enhancing the osteogenic signals exerted by vascular smooth muscle cells. Biochem Biophys Res Commun. 2012;425(1):39-44. doi:10.1016/j.bbrc.2012.07.045

29. New SE, Aikawa E. Role of extracellular vesicles in de novo mineralization: an additional novel mechanism of cardiovascular calcification. Arterioscler Thromb Vasc Biol. 2013;33(8):1753-1758. doi:10.1161/ATVBAHA.112.300128

30. Rumberger JA, Simons DB, Fitzpatrick LA, Sheedy PF, Schwartz RS. Coronary artery calcium area by electron-beam computed tomography and coronary atherosclerotic plaque area. A histopathologic correlative study. Circulation. 1995;92 (8):2157-2162. doi:10.1161/01.CIR.92.8.2157

31. Proudfoot D, Shanahan CM, Weissberg PL. Vascular calcification: new insights into an old problem. J Pathol. 1998;185(1):1-3. doi:10.1002/ (SICI)1096-9896(199805)185:1<1::AID-PATH89>3.0.CO;2-J

32. Lee RT, Grodzinsky AJ, Frank EH, Kamm RD, Schoen FJ. Structuredependent dynamic mechanical behavior of fibrous caps from human atherosclerotic plaques. Circulation. 1991;83(5):1764-1770. doi:10.1161/01.CIR.83.5.1764

33. Richardson PD, Davies MJ, Born GV. Influence of plaque configuration and stress distribution on fissuring of coronary atherosclerotic plaques. Lancet (London, England). 1989;2(8669):941-944. doi:10.1016/S0140-6736(89)90953-7

34. Chai CK, Akyildiz AC, Speelman L, et al. Local axial compressive mechanical properties of human carotid atherosclerotic plaques-characterisation by indentation test and inverse finite element analysis. $J$ Biomech. 2013;46(10):1759-1766. doi:10.1016/j. jbiomech.2013.03.017

35. Matsuo Y, Takumi T, Mathew V, et al. Plaque characteristics and arterial remodeling in coronary and peripheral arterial systems. Atherosclerosis. 2012;223(2):365-371. doi:10.1016/j. atherosclerosis.2012.05.023 
36. Winter PM, Caruthers SD, Zhang H, Williams TA, Wickline SA, Lanza GM. Antiangiogenic synergism of integrin-targeted fumagillin nanoparticles and atorvastatin in atherosclerosis. JACC Cardiovasc Imaging. 2008;1(5):624-634. doi:10.1016/j.jcmg.2008. 06.003

37. Takaya N, Yuan C, Chu B, et al. Association between carotid plaque characteristics and subsequent ischemic cerebrovascular events: a prospective assessment with MRI--initial results. Stroke. 2006;37 (3):818-823. doi:10.1161/01.STR.0000204638.91099.91

38. Yang J, Pan X, Zhang B, et al. Superficial and multiple calcifications and ulceration associate with intraplaque hemorrhage in the carotid atherosclerotic plaque. Eur Radiol. 2018;28(12):4968-4977. doi:10.1007/s00330-018-5535-7

39. Teng Z, He J, Degnan AJ, et al. Critical mechanical conditions around neovessels in carotid atherosclerotic plaque may promote intraplaque hemorrhage. Atherosclerosis. 2012;223(2):321-326. doi:10.1016/j.atherosclerosis.2012.06.015
40. Nandalur KR, Hardie AD, Raghavan P, Schipper MJ, Baskurt E, Kramer CM. Composition of the stable carotid plaque: insights from a multidetector computed tomography study of plaque volume. Stroke. 2007;38(3):935-940. doi:10.1161/01.STR.0000257995.74834.92

41. Gennaro G, Ménard C, Giasson E, et al. Role of p44/p42 MAP kinase in the age-dependent increase in vascular smooth muscle cell proliferation and neointimal formation. Arterioscler Thromb Vasc Biol. 2003;23(2):204-210. doi:10.1161/01.ATV.0000053182.58636.BE

42. Kockx MM, De Meyer GR, Muhring J, Jacob W, Bult H, Herman AG. Apoptosis and related proteins in different stages of human atherosclerotic plaques. Circulation. 1998;97(23):2307-2315. doi:10.1161/01.CIR.97.23.2307

43. van den Bouwhuijsen QJ, Vernooij MW, Hofman A, Krestin GP, van der Lugt A, Witteman JC. Determinants of magnetic resonance imaging detected carotid plaque components: the Rotterdam Study. Eur Heart J. 2012;33(2):221-229. doi:10.1093/eurheartj/ehr227

\section{Publish your work in this journal}

Therapeutics and Clinical Risk Management is an international, peerreviewed journal of clinical therapeutics and risk management, focusing on concise rapid reporting of clinical studies in all therapeutic areas, outcomes, safety, and programs for the effective, safe, and sustained use of medicines. This journal is indexed on PubMed Central, CAS,
EMBase, Scopus and the Elsevier Bibliographic databases. The manuscript management system is completely online and includes a very quick and fair peer-review system, which is all easy to use. Visit http://www.dovepress.com/testimonials.php to read real quotes from published authors. 